\title{
Tratamiento farmacológico: la desintoxicación
}

\author{
Soler, P.A.; Guasch, M.
}

Servicio Psiquiatría. Hospital Mútua de Terrassa. (Barcelona)

\section{RESUMEN}

El síndrome de abstinencia alcohólico se produce por la disminución brusca de la alcoholemia en individuos con neuroadaptación al alcohol. La deprivación comporta alteraciones en diversos sistemas de neurotransmisores, provocando una hiperactivación noradrenérgica, un incremento de la sensibilidad del receptor NMDA y una inhibición del GABA.

El síndrome de abstinencia alcohólica puede objetivarse mediante diversas escalas (Ciwa, Soler Insa), que permiten valorar la intensidad de la abstinencia. Las pautas de tratamiento más contrastadas se ajustan en función de la severidad del cuadro. Asimismo, se exponen las pautas fijas, también utilizadas y publicadas, pero para los autores menos aconsejables.

El clometiazol, el tetrabamato y las benzodiacepinas son los fármacos más utilizados y sobre los que existen más datos de eficacia y seguridad, pero también se detallan algunos estudios con otros fármacos como la carbamazepina y otros anticonvulsivantes, los beta-bloqueadores, los antagonistas alfa2, los antagonistas del calcio y otros.

Finalmente se dan recomendaciones terapéuticas para casos especiales como el delirium tremens, las convulsiones durante la abstinencia o los casos de la mujer embarazada, el anciano o el paciente postquirúrgico.

Palabras clave: abstinencia del alcohol, valoración, intensidad, tratamiento, farmacoterapia.

\begin{abstract}
Alcoholic abstinence syndrome is produced by the abrupt reduction of alcoholism in individuals with neuroadaptation to alcohol. Deprivation leads to alterations in diverse neurotransmitter systems, provoking a noradrenergic hyperactivity, an increase in NMDA receptor sensitivity and GABA inhibition.

Alcohol abstinence syndrome may be objectified through several scales (Ciwa, Soler Insa) that enable the intensity of abstinence to be assessed. The more contrasted treatment guidelines are adapted in accordance with the severity of the case. In addition, the set guidelines are given which are also utilised and published, but by less reliable authors.

Chlormethiazole, tetrabamate and the benzodiazepines are the most used drugs and the ones on which more data exists on efficacy and safety but details are given of certain studies with other drugs such as carbamazephine and other anticonvulsivants, the beta-blockers, the alpha2 antagonists, the calcium antagonists and others.

Lastly, therapeutic recommendations are given for special cases such as delirium tremens, convulsions during abstinence, cases of pregnant women, the old or the post operational patient.
\end{abstract}

Key words: alcohol abstinence, evaluation, intensity, treatment pharmacotherapy.
$\mathbf{L}$ a dependencia del alcohol es un grave problema sanitario. La mayoría de los estudios estima que el riesgo a lo largo de la vida de padecer dependencia del alcohol oscila entre el $10 \%$ para los varones y el $3-5 \%$ para las mujeres, y el riesgo de alcanzar un consumo perjudicial prácticamente el doble. La dependencia del alcohol conlleva una elevada comorbilidad médica y psiquiátrica, oscilando la prevalencia de los alcohólicos visitados en la asistencia primaria entre el $15 \%$ y el $50 \%$ según los autores ${ }^{1}$. Los estudios sobre prevalencia de problemas relacionados con el alcohol en los servicios de 
medicina de los hospitales generales en España dan cifras entre el $15 \%$ y el $35 \%^{2}$. Los profesionales de todas las áreas de la medicina se encuentran con cierta frecuencia con el problema del manejo del síndrome de abstinencia del alcohol, ya que la hospitalización o el embarazo por ejemplo, son a menudo los precipitantes de una supresión brusca del alcohol ${ }^{3}$.

\section{SÍNDROME DE ABSTINENCIA ALCOHÓ- LICA}

La sintomatología del síndrome de abstinencia alcohólica (SAA), ha sido descrita desde hace siglos. Victor y Adams en 1953 describen detalladamente el síndrome de abstinencia del alcohol en una serie de pacientes admitidos en una unidad especializada en EEUU, con los síntomas que reconocemos en la actualidad: temblor, náuseas, ansiedad, diaforesis, tinnitus, calambres musculares, convulsiones, alucinaciones y delirium $^{4}$. En 1992, en la última revisión de la Clasificación Estadística Internacional de enfermedades y problemas de la salud (CIE 10) es tipificado como:

-F10.30. Síndrome de abstinencia no complicado.

-F10.31. Síndrome de abstinencia con convulsiones.

-F10.40. Síndrome de abstinencia con delirium, sin convulsiones.

-F10.41. Síndrome de abstinencia con delirium, con convulsiones.

El Síndrome de abstinencia se da en el individuo con neuroadaptación al alcohol y se relaciona con el descenso del nivel de alcohol en sangre y los consecuentes cambios que esto produce en el sistema nervioso central (SNC). La sintomatología depende de diversos factores como el grado de dependencia, la tolerancia metabólica, la dosis diaria, el tiempo de consumo y también probablemente de la presencia de factores genéticos predisponentes. La aparición mas o menos aguda o retardada en el tiempo depende de la curva de descenso de la alcoholemia, pudiendo objetivarse entre las 4-12 horas y hasta los 3-4 días siguientes a la supresión del tóxico, siendo la duración del SAA de 5-10 días. Son menos frecuentes los casos de síndromes de abstinencia prolongada que perduran hasta dos semanas. En un estudio comunitario realizado en Australia se objetivó que el $14 \%$ de los "bebedores" presentaban sudoración el día después de consumir alcohol, y que el $9 \%$ tenían temblor al despertar$\mathrm{se}^{5}$. EI SAA no es infrecuente en pacientes ingresados. Un estudio reciente realizado también en Australia en una muestra de 2046 pacientes ingresados demostró que un $8 \%$ de los mismos presentaron sintomatología de abstinencia al alcohol, y de éstos un $8 \%$ (un $0,65 \%$ del total de la muestra) presentaban convulsiones o delirium ${ }^{6}$.

\section{Fisiopatología del síndrome de abstinen- cia al alcohol.}

Para conocer la fisiopatología del SAA es necesario hacer una somera referencia a los efectos neuroquímicos que produce el alcohol tanto en su administración aguda como crónica. Los estudios de mayor relevancia e interés se refieren a aminoácidos inhibidores (GABA), excitatorios (glutamato), dopamina, serotonina, opiáceos endógenos, noradrenalina y acetilcolina ${ }^{7}$. El etanol actúa sobre los receptores GABAA y GABAB. El GABA es el principal neurotransmisor inhibidor de todo el SNC. De forma aguda, el alcohol facilita la transmisión GABAérgica, abriendo el paso de cloro por el canal del receptor GABAA y su efecto crónico es el contrario produciendo una disminución en la expresión de la subunidad del receptor GABAA. Este efecto facilitador condicionaría en parte el refuerzo positivo al alcohol. El receptor GABAв está implicado en otros efectos del etanol como la hipotermia y las alteraciones de la motricidad. Durante la abstinencia al alcohol el complejo del receptor GABA se ve privado del efecto del alcohol sobre el flujo de cloro, con el consiguiente decremento de la función del 
GABA, lo que provoca desinhibición y parte de los síntomas propios del SAA.

La administración de alcohol antagoniza el efecto de otro neurotransmisor excitatorio, el glutamato. Los receptores NMDA (N-Metil-DAspartato) del glutamato están ampliamente distribuidos en el SNC y tienen un importante papel en el desarrollo neuronal y en la plasticidad sináptica, pero la sobreestimulación de estos receptores puede producir neurotoxici$\mathrm{dad}^{8}$. Constituyen un importante mediador en la acción del etanol. El alcohol es un potente inhibidor de los agonistas del receptor NMDA, provocando su consumo crónico un aumento de la función del glutamato, incrementos del calcio intracelular y la muerte celular, lo que parece relacionarse con los efectos destructivos del alcohol sobre la memoria y el aprendizaje. Durante la abstinencia del alcohol se produce un incremento en la sensibilidad del receptor NMDA.

Se han demostrado efectos de la administración crónica sobre los receptores $5-\mathrm{HT}_{1 \mathrm{~A}} \mathrm{y}$

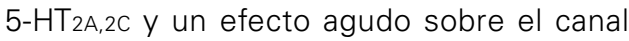
iónico del 5-HT3. En el síndrome de abstinencia se produce un descenso de los niveles de serotonina. Se cree que el sistema serotoninérgico actúa como mediador entre el etanol y el sistema dopaminérgico. La estimulación vía receptor $5-\mathrm{HT}_{3}$ incrementaría la liberación de dopamina (DA) del núcleo accumbens, lo que también está implicado en los efectos reforzadores del alcohol. El alcohol tiene un efecto dopaminérgico importante, provocando de manera aguda la liberación de la DA del núcleo accumbens. La administración crónica del alcohol provoca por el contrario una deplección de DA. Se acepta que los efectos del etanol sobre este sistema son similares a los de cualquier otra droga adictiva, y están relacionados con los efectos gratificantes y reforzadores $^{9}$. Se postula que este efecto reforzante final es debido al incremento de la transmisión dopaminérgica, aunque mediatizado por los receptores opiáceos $\delta, \mu$ o $a_{m b o s}{ }^{10}$. Sobre la noradrenalina también se ha observado un incremento de la transmisión a dosis bajas y un efecto inhibidor a dosis altas, principalmente en el locus coeruleus. Durante el SAA se produce un incremento de la transmisión noradrenérgica. Aparecen signos de hiperactividad del sistema nervioso simpático. Este incremento de actividad se ha objetivado mediante estudios que demuestran la elevación de los niveles de noradrenalina y su metabolito 3 metoxi, 4 hidroxi, feniletilenglicol (MHPG) durante el SAA. El nivel basal de MHPG se ha relacionado con el efecto kindling, de forma que a mayor número de deprivaciones sucesivas mayor es el nivel de MHPG aumentando con ello la intensidad del síndrome de abstinencia" ${ }^{11}$.

Sobre la acetilcolina se han descrito importantes reducciones de la actividad de la enzima colin-acetil-transferasa, necesaria para la síntesis de acetilcolina en diversas áreas cerebrales en alcohólicos de larga evolución. También se han descrito cambios en los receptores de acetilcolina talámicos en cuanto a su número.

El alcoholismo crónico se acompaña frecuentemente de cambios en el eje hipotálamo-hipofiso-adrenal (HPA). Los pacientes con alcoholismo frecuentemente presentan una no supresión en el test de la dexametasona y un pobre incremento de la ACTH tras la inyección de $\mathrm{CRH}^{12}$. Se produce una activación del sistema HPA durante el síndrome de abstinencia al alcohol ${ }^{13}$. Las elevaciones en los niveles de corticoesteroides se asocian con alteraciones del humor y deterioro cognitivo. Otros síntomas como debilidad, hipertensión, confusión mental y depresión también pueden deberse parcialmente a la elevación de los glucocorticoides observada durante el SAA. También puede observarse un incremento de la hormona antidiurética y de la aldosterona.

\section{El síndrome de abstinencia al alcohol leve- moderado}

La clínica del síndrome de abstinencia se inicia entre las 6 y las 8 horas tras suprimir el consumo de alcohol y se caracteriza por "craving" o apetencia por el consumo, temblor fino distal, sudoración, náuseas o vómitos, 
xerostomía, ansiedad y cefaleas. Se incrementan la tensión arterial y la frecuencia cardíaca, y pueden existir midriasis, hiperventilación e hipertermia. Son frecuentes las alteraciones psíquicas principalmente ansiedad y cambios de humor. En los casos más leves el SAA puede remitir espontáneamente, incluso sin tratamiento de ningún tipo; pero debe tenerse en cuenta que también es posible que a partir de las 24-48 horas de abstinencia los síntomas aumenten en intensidad.

\section{Las convulsiones en la abstinencia al alco- hol ("Rum Fits")}

Se presentan en el 5\%-15\% de los casos, entre las 6-8 horas de supresión del alcohol y hasta las 48 horas (con un pico máximo entre las 12-24 horas), observándose con mayor frecuencia en los individuos con grave dependencia al alcohol, en aquellos en que además existe una dependencia mixta a barbitúricos o benzodiacepinas, y en los casos en que existen antecedentes de convulsiones tanto secundarias a la supresión de alcohol, como en los afectos de una enfermedad epilépti$\mathrm{ca}^{14}$. Clínicamente se manifiestan en forma de convulsiones tónico-clónicas generalizadas. La presencia de una crisis parcial ha de poner en duda el diagnóstico, e indica que es necesaria una evaluación neurológica completa, descartando principalmente la existencia de un traumatismo craneoencefálico. Las convulsiones del SAA evolucionan de forma favorable, sin secuelas. Si las convulsiones perduran más de una semana se ha de sospechar una enfermedad médica concomitante o el uso de medicaciones que disminuyan el umbral convulsivo ${ }^{15}$.

\section{Síndrome de abstinencia al alcohol con delirium}

El síndrome de abstinencia con delirium afecta a menos de un $5 \%$ de los pacientes con síndrome de abstinencia y hasta un 30\% de los pacientes que han presentado un síndrome de abstinencia con convulsiones. En un reciente estudio retrospectivo realizado en EEUU se observó que el $24 \%$ de 200 pacientes con dependencia del alcohol ingresados en un hospital americano desarrollaron un delirium tremens, lo que relacionaron con el hecho de que los pacientes presentaran una o más enfermedades intercurrentes o con el hecho de no haber consumido alcohol en los 2 días previos al ingreso ${ }^{16}$. Se presenta con máxima intensidad entre las 48 y 72 horas, en personas con grave dependencia y tiempo prolongado de consumo, más frecuentemente cuando son hospitalizadas o encamadas en su domicilio. Es un cuadro grave tóxicoconfusional que requiere ingreso hospitalario urgente y que presenta una mortalidad sin tratamiento del $20 \%$ debido a las complicaciones metabólicas y enfermedades médicas concomitantes, como alteraciones hidroelectrolíticas y del equilibrio ácido-base, infecciones intercurrentes o enfermedades cardiovasculares.

Para su diagnóstico es imprescindible la presencia de disminución del nivel de conciencia, aunque con respuesta a estímulos. Puede ser fluctuante o manifestarse de forma constante, pudiendo evolucionar al coma. También resultan imprescindibles para el diagnóstico la desorientación temporoespacial, que al inicio puede ser de predominio nocturno y los trastornos sensoperceptivos en forma de alucinaciones visuales principalmente (formas filamentosas, luces o microzoopsias) que se acompañan de un estado de ansiedad importante, no siendo infrecuentes las alucinaciones auditivas.

Existen también importantes trastornos mnésicos y falsos reconocimientos. Pueden aparecer ideas delirantes autorreferenciales o el clásico delirio ocupacional. El enfermo acostumbra a presentar un estado de agitación psicomotriz con insomnio total o inversión del ciclo sueño-vigilia, aunque en algunos casos puede presentarse disminución de la actividad. Signos y síntomas de hiperactividad autonómica, como diaforesis importante que puede provocar deshidratacion, hipertermia, hipertensión y taquicardia y sintomatología neurológica, como temblor generalizado y 
palpable, disartria y marcha inestable, con incoordinación motora.

Debe comentarse que existe una controversia importante en relación con las alucinosis alcohólicas, ya que en el DSM IV se acepta la posibilidad de que aparezcan después de la supresión del alcohol, pero la CIE-10 indica que aparecen durante el consumo o más raramente inmediatamente después de él. Es más frecuente en varones (4/1), caracterizándose por la presencia de alucinaciones auditivas de tono amenazante, que son vividas con gran ansiedad por el paciente. Pueden durar algunos días, pero es más frecuente su carácter subagudo, evolucionando en un $10 \%$ hacia una forma crónica. En la alucinosis no se altera el nivel de conciencia y no existe sintomatología de abstinencia.

\section{Valoración de la intensidad del síndrome de abstinencia.}

Las mediciones cuantitativas constituyen una parte imprescindible en la valoración del síndrome de abstinencia al alcohol y también son útiles para realizar comparaciones de las poblaciones en estudio. Es, sin embargo, difícil para los investigadores y profesionales de la salud encontrar una medición que sea fiable, validada y ampliamente utilizada, habiéndose utilizado diferentes escalas para este uso. Una escala ideal es aquella que "ayude al diagnóstico del síndrome de abstinencia al alcohol, indique la necesidad de tratamiento farmacológico, alerte al personal sanitario de la presencia de un síndrome de abstinencia severo, e indique en qué momento puede ser reducido o suspendido el tratamiento farma-

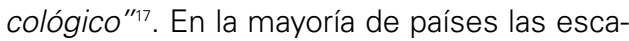
las más utilizadas son la TSA (Total Severity Assessment) y sobretodo la CIWA (Clinical Institute Withdrawal Assessment Scale for Alcohol), y de ellas se derivan gran parte del resto de mediciones. Son la CIWA y sus derivaciones (la CIWA-Ar, sobretodo) las más utilizadas, sin lugar a dudas, para la valoración de la abstinencia y se han usado tanto en el manejo clínico como en investigación. Puede ser fácilmente aplicada por el personal sanita- rio, tanto médico como de enfermería. En nuestro país, la escala de Soler PA y cols. realizada en 1981, consta de 11 ítems que puntúan entre 0 y 3 , pudiendo alcanzar una valoración máxima de 33 puntos (Ver Tabla I). Debe aplicarse una de estas escalas a todos los pacientes que están realizando una desintoxicación del alcohol. La CIWA consta de 15 ítems (náuseas, temblor, sudoración, trastornos del tacto, auditivos y visuales, alucinaciones, orientación, contacto, ansiedad, trastornos del pensamiento, convulsiones, cefaleas y enrojecimiento facial) (Ver Tabla II). La CIWA-Ar, versión revisada de la CIWA, constituye el "patrón oro" por ser sencilla y rápida a la hora de ser aplicada y su uso está muy extendido. Consta de 10 ítems (nauseas, temblor, sudoración, alteraciones del tacto, auditivas y visuales, ansiedad, orientación, convulsiones y cefaleas), que puntúan entre 0 y 7 , a excepción del parámetro orientación que puntúa de 0-4. Se considera que si el paciente presenta una puntuación por debajo de 8-10 no se requiere utilizar tratamiento farmacológico, siendo el síndrome de abstinencia muy severo cuando la puntuación es de 20 o más.

Recordar que el DSM-IV indica como signos y síntomas de SAA: hiperactividad autonómica (sudoración o pulso arterial superior a 100), temblor, insomnio, náuseas o vómitos, alucinaciones o ilusiones visuales, táctiles o auditivas, agitación psicomotriz, ansiedad y convulsiones. Estos parámetros pueden ser utilizados para valorar la intensidad de la abstinencia (número de síntomas o signos presentes o intensidad de cada uno de ellos).

\section{PREVENCIÓN Y TRATAMIENTO DEL SIN- DROME DE ABSTINENCIA AL ALCOHOL}

La necesidad de realizar un tratamiento óptimo del síndrome de abstinencia radica en mejorar el confort del paciente, en prevenir la aparición de complicaciones y en facilitarnos iniciar un tratamiento rehabilitador a largo plazo $^{18}$. Para algunos autores sigue siendo 
Tabla I. Valoración de la intensidad de la abstinencia alcohólica

\begin{tabular}{|c|c|c|c|c|}
\hline Síntomas & 0 puntos & 1 punto & 2 puntos & 3 puntos \\
\hline Ansiedad & No & + & ++ & +++ \\
\hline Sudación & No & Solo nocturna & Nocturna y diurna & Gran diaforesis \\
\hline Temblor & $\begin{array}{c}\text { No o fino distal y } \\
\text { matutino }\end{array}$ & $\begin{array}{l}\text { Fino distal } \\
\text { permanente }\end{array}$ & $\begin{array}{c}\text { Intenso permanente } \\
\text { en lengua }\end{array}$ & $\begin{array}{c}\text { Generalizado } \\
\text { y palpable }\end{array}$ \\
\hline $\begin{array}{l}\text { Déficit de } \\
\text { conciencia }\end{array}$ & No & $\begin{array}{l}\text { Episodios nocturnos } \\
\text { aislados }\end{array}$ & Nocturnos y diurnos & $\begin{array}{l}\text { Notorio y } \\
\text { constante }\end{array}$ \\
\hline Desorientación & No & Episodios aislados & Desorientación total & $\begin{array}{l}\text { Desorientación } \\
\text { permanente }\end{array}$ \\
\hline $\begin{array}{l}\text { Trastornos de } \\
\text { la percepción }\end{array}$ & No & Sólo nocturnos & Nocturnos y diurnos & $\begin{array}{c}\text { Alucinaciones } \\
\text { constantes y falsos } \\
\text { reconocimientos }\end{array}$ \\
\hline $\begin{array}{l}\text { Trastornos de } \\
\text { la memoria }\end{array}$ & No & $\begin{array}{c}\text { Amnesias referidas a } \\
\text { la noche }\end{array}$ & $\begin{array}{c}\text { Amnesias referidas al } \\
\text { día y la noche }\end{array}$ & $\begin{array}{c}\text { Amnesias de más } \\
\text { de } 12 \text { horas }\end{array}$ \\
\hline Insomnio & No & $\begin{array}{l}\text { De conciliación o } \\
\text { despertar precoz }\end{array}$ & Intermitente & Total \\
\hline Hipertermia & No & No & No & Sí \\
\hline $\begin{array}{l}\text { Actividad } \\
\text { psicomotriz }\end{array}$ & Normal & Normal & Inquietud & Agitación \\
\hline $\begin{array}{l}\text { Delirio } \\
\text { ocupacional }\end{array}$ & No & No & No & Sí \\
\hline
\end{tabular}

Soler P. A. y cols, 1981.

Intensidad de la abstinencia alcohólica: Grado 1: de 0 a 7 puntos. Grado 2: de 8 a 15 puntos. Grado 3: de 16 a 23 puntos. Grado 4: más de 24 puntos.

controvertido en qué casos está indicado un abordaje farmacológico y en general, cuál es el tratamiento más apropiado en cada caso, teniendo en cuenta que entre un $13 \%$ y un $71 \%$ de los pacientes que realizan una desintoxicación del alcohol experimentarán sintomatología de abstinencia de forma evidente ${ }^{19}$. La severidad del síndrome de abstinencia y la probabilidad de aparición de complicaciones vendrán determinados por la historia de consumo de alcohol, sobretodo si han habido abusos de alcohol importantes en los días antes del ingreso, la severidad de la dependencia, el número de desintoxicaciones previas y posiblemente también la historia familiar de alcoholismo ${ }^{20}$.

Es imprescindible practicar a todos los pacientes que vayan a realizar una desintoxicación del alcohol un examen físico, y una analítica que incluya bioquímica con glucosa, ionograma, parámetros de función renal y hepática, hemograma, pruebas de coagulación y sedimento urinario. Hay que descartar la presencia de alteraciones cardiacas, patología hepática o pancreática, infecciones concomitantes, sangrado gastrointestinal o enfermedades neurológicas o psiquiátricas concomitantes. En primer lugar debe estabilizarse al sujeto, corrigiendo las posibles alteraciones hidroelectrolíticas, que se presentan frecuentemente en los sujetos con consumos crónicos de alcohol, e incluyen hipomagnesemia, hipofosfatemia e hipokaliemia. En estos pacientes son comunes las deficiencias nutricionales y vitamínicas, por lo que se debe administrar vitamina B1 y B6, y ácido fólico en la mayoría de los casos. La administración de ácido fólico ha de ser prudente dado que si existe un déficit de vitamina B12 
Tabla II. Valoración del síndrome de abstinencia alcohólico.

\section{Náuseas y vómitos}

0 . Ausencia de náuseas y vómitos

1. Náuseas moderadas sin vómitos

4. Náuseas intermitentes con arcadas secas

7. Náuseas constantes con arcadas y vómitos

\section{Temblor (brazos extendidos y dedos separados)}

0 . Ningún temblor

1. No visible, pero sensación de temblor fino

4. Temblor moderado con los brazos extendidos

7. Temblor grave incluso sin los brazos extendidos

\section{Sudación paroxística}

0 . Ausencia de sudación

1. Sudación discreta. Palmas de manos húmedas

4. Sudación evidente en facies

7. Sudación profusa

Trastornos del tacto (hormigueo, picor, entumecimiento)

0 . Ausentes

3. Sensaciones táctiles moderadas

4 Presencia de alucinaciones táctiles moderadas

6. Alucinaciones tactiles graves

Trastornos auditivos (percepción de sonidos)

0 . Ausentes

1. Muy discretos

3. Sensaciones auditivas moderadas

4. Presencia de alucinaciones auditivas

6. Alucinaciones auditivas graves

\section{Trastornos visuales}

0 . Ausentes

1. Muy discretos

3. Sensaciones visuales moderadas

4. Presencia de alucinaciones visuales

6. Alucinaciones visuales graves

\section{Alucinaciones}

0 . Ninguna

1. Solo auditivas o visuales

2. Auditivas y visuales incongruentes

3. Auditivas y visuales congruentes

\section{Orientación autopsíquica y alopsíquica}

0 . Orientado. Puede añadir algunas referencias

1. Dudoso con respecto a algunos datos (fecha, dirección)
2. Desorientado en tiempo (no más de 2 días)

3. Desorientado en tiempo (más de 2 días)

4. Desorientación espacial y/o en persona

\section{Calidad del contacto}

0 . Sintónico con el examinador

1. Tiende a distraerse

2. Parece contactar, pero está desconectado del medio ambiente

4. Periódicamente parece indiferente

7. No establece ningún contacto con el examinador

\section{Ansiedad}

0 . Ausente

1. Ansiedad discreta

4. Ansiedad moderada

7. Equivalente a los estados de pánico observados en delirium

Trastornos del pensamiento (fuga de ideas)

0 . Ningún trastorno

1. Alguna pérdida del control de los contenidos del pensamiento

2. Contenidos desagradables de forma continuada

3. Fuga e incoherencia del pensamiento

\section{Convulsiones}

0 . No

1. Sí

Cefaleas y sensación de plenitud cefálica

0 . Ausente

1. Muy discreta

2. Discreta

3. Moderada

4. Entre moderada y grave

5. Grave

6. Muy grave

7. Extremadamente grave

Enrojecimiento facial

0 . Ninguno

1. Moderado

2. Grave

TOTAL PUNTUACION

Clinical Institute Withdrawal Assessment (CIWA). 
puede producirse una degeneración combinada de la médula, motivo por el que deben darse conjuntamente. Es importante recordar que la administración de tiamina debe ser previa a la de glucosa, ya que en el caso de hipoglucemia la administración de glucosa puede producir una deplección de tiamina lo que puede aumentar el riesgo de encefalopatía de Wernicke.

$\mathrm{Ha}$ de realizarse una detallada entrevista clínica no solo al paciente, si no también a familiares y/o allegados, incluyendo el tiempo de evolución y el patrón de consumo, la cantidad diaria de alcohol ingerida, si ha habido intentos de desintoxicación previos y las posibles complicaciones aparecidas (síntomas de abstinencia importantes, delirium, convulsiones). Se hará especial énfasis en las dosis ingeridas en los días previos a la desintoxicación, porque en caso de consumos elevados se planteará de entrada realizar un abordaje farmacológico en la mayoría de los casos. Será necesario investigar consumo concomitante de otros fármacos o drogas, especialmente aquellos con tolerancia cruzada con el alcohol. Una historia de sintomatología de abstinencia en el pasado, múltiples desintoxicaciones previas, o una ingesta importante de alcohol en los últimos días, predicen el riesgo de presentar un severo síndrome de abstinencia. Todos los pacientes con historia de convulsiones deberán recibir tratamiento preventivo de manera sistemática.

El tratamiento de la desintoxicación del alcohol puede ser igualmente seguro y eficaz tanto si se realiza de forma ambulatoria como mediante un ingreso hospitalario, siempre que se realice una selección adecuada de los pacientes. Aproximadamente un $70 \%$ de los pacientes que comienzan un programa de desintoxicación ambulatoria lo finalizan con éxito (35\%-95\%), y un 50\% continúan un tratamiento de rehabilitación, según la mayoría de trabajos revisados. Entre un $10 \%$ y un $20 \%$ de los pacientes requieren ser ingresados $^{21}$. En general se consideran criterios de ingreso hospitalario, que el paciente presente síntomas de abstinencia severos, es decir, más de 20 puntos en la escala CIWA-Ar o más de 10 en la escala de Soler y cols., que exista patología médica o psiquiátrica concomitante, historia de síndrome de abstinencia importante, convulsiones o delirium tremens, trastorno por dependencia o abuso de otras sustancias, existencia de factores sociales desfavorables y/o escaso apoyo familiar, bajo coeficiente intelectual o falta de cooperación.

\section{Tratamiento de la abstinencia de alcohol leve-moderada}

Aunque no existen dudas acerca de que se ha de realizar tratamiento farmacológico en todos aquellos pacientes con sintomatología severa de abstinencia, sí existe mayor controversia en el caso de sintomatología leve o moderada. En varios estudios se ha descrito el tratamiento ambulatorio satisfactorio de los pacientes con síntomas de abstinencia de leves a moderados $22,23,24,25$. En nuestro país está extendido el uso de la farmacoterapia, pero en otros lugares se considera que la hidratación, un correcto aporte nutricional, la reducción de estímulos sensoriales y el apoyo al paciente son suficientes en el $75 \%$ de los $\operatorname{casos}^{26}$. Sin embargo, cabe señalar que diversos estudios han demostrado que durante el síndrome de abstinencia se produce un incremento de la neurotransmisión excitatoria y de la actividad corticoidea, ambos tóxicos para las células nerviosas, induciendo un fenómeno de "kindling", es decir incrementando la sensibilización neuronal, de manera que hay que ser prudentes a la hora de considerar dejar sin tratamiento a los pacientes que han experimentado abstinencias repetidas ya que se pueden producir en el futuro síndromes de abstinencia de mayor gravedad y posibles alteraciones permanentes del SNC. En aquellos pacientes con enfermedades médicas concomitantes, el tratamiento farmacológico debe ser administrado aún en caso de que la sintomatología sea de grado leve o moderado, así como en el caso de que exista historia de crisis convulsivas. En nuestra opinión ha de realizarse tratamiento farmacológico siempre, ya que se dispone de un arsenal terapéutico que 
administrado de forma prudente e individualizado para cada paciente tiene una gran eficacia y un amplio margen de seguridad.

Se han investigado aproximadamente unas 150 sustancias para el tratamiento del síndrome de abstinencia alcohólico, existiendo diferencias significativas entre los diferentes profesionales sobre cuáles constituyen el tratamiento y las dosis óptimas ${ }^{27}$. En la mayoría de revisiones y meta-análisis realizados se ha concluido que no hay ningún tratamiento único que pueda considerarse superior a los otros. La selección depende del criterio médico y de otros factores del paciente, como la edad y la presencia de enfermedades médicas, principalmente hepáticas o respiratorias. En cualquier caso el tratamiento ideal en el SAA sería aquel con un inicio de acción rápido, con una larga duración en su acción, con un amplio margen de seguridad, un metabolismo no dependiente de la función hepática y con bajo potencial de abuso.

Las mediciones cuantitativas de la intensidad del SAA son una parte necesaria del control clínico y facilitan criterios objetivos que permiten al clínico el optar o no por un tratamiento farmacológico, debiendo administrarse tratamiento farmacológico a todos los pacientes admitidos para una desintoxicación si la puntuación en la escala CIWA-Ar es superior a 8-10. Por otra parte la mayor o menor puntuación en las escalas que valoran la intensidad de la sintomatología de abstinencia decidirán para cada paciente cuál es la dosis ideal de fármacos con tolerancia cruzada con el alcohol a utilizar, lo que reducirá la administración de medicación de forma innecesaria.

Los fármacos más utilizados para el tratamiento del SAA son aquellos que presentan una tolerancia cruzada con el alcohol, como las benzodiacepinas, el clormetiazol y el tetrabamato.

Siempre, e independientemente de la intensidad de la abstinencia, se recomienda administrar 100 mg./día de tiamina via i.m. durante una semana aproximadamente, para prevenir la encefalopatía de Wernicke, no existiendo ninguna contraindicación para su administración.

\section{Edsilato de Clormetiazol}

En nuestro medio, no en EEUU, el Edsilato de Clormetiazol es quizás el fármaco más utilizado para el tratamiento del síndrome de abstinencia. Constituye la fracción tiazólica de la vitamina B1 y se encuentra disponible para su administración oral o parenteral. Comenzó a utilizarse en el tratamiento del delirium tremens en 1957, y se introdujo en el tratamiento de los síntomas moderados de la abstinencia en $1965^{28}$. Tiene una acción ansiolítica, antitremórica, hipnótica y anticonvulsiva. La intolerancia al clormetiazol, aunque poco frecuente, se manifiesta de forma inmediata en forma de congestión, cosquilleo nasal, rinorrea, estornudos, prurito, rubefacción facial, xerostomía y tos. Tiene un riesgo de potencial de abuso y de depresión respiratoria. Especial atención merecen en consecuencia los pacientes con enfermedad respiratoria. La dosis más utilizada oscila, en función de la intensidad de la abstinencia, entre 7 y 16 comprimidos de $192 \mathrm{mg}$./día, repartidos en 34 tomas diarias, debiendo reducirse lentamente la dosificación a ritmo de un comprimido al día aproximadamente, reservando siempre una dosis superior nocturna. Se recomiendan pautas que no sobrepasen las dos semanas de tratamiento (Tabla III). Especial atención merecen los pacientes con enfermedad respiratoria, ya que el clormetiazol puede provocar depresión respiratoria e hipotensión. Es posible su administración parenteral, lo que es especialmente útil en los cuadros de abstinencia grave con delirium. Si existe agitación pueden administrarse $150 \mathrm{ml}$. de Clormetiazol a ritmo de hasta 100 microgotas/minuto, procurando conseguir un sueño superficial, y estableciendo posteriormente una dosis de mantenimiento que puede oscilar entre $10 \mathrm{y}$ 20 microgotas/minuto, dependiendo de la intensidad de la sintomatología. No se recomienda superar la dosis de un vial (4 gramos) en 24 horas. Ha de instaurarse en cuanto sea posible la administración oral. 
Tabla III. Pautas de clometiazoIPAUTAS DE CLOMETIAZOL ${ }^{62}$

\begin{tabular}{lcccc}
\hline Día & Pauta 1 & Pauta 2 & Pauta 3 & Pauta 4 \\
\hline 1 & $2-2-3$ & $3-3-4$ & $4-4-4-4-$ & Parenteral: Administrar un vial (4 g) \\
2 & $2-2-2$ & $3-3-3$ & $3-4-3-4$ & en 24 horas (24 microgotas/min., aprox.) \\
3 & $1-2-2$ & $3-2-3$ & $3-3-4$ & con sueroterapia conjunta. \\
4 & $1-1-2$ & $2-2-3$ & $3-3-3$ & \\
5 & $1-1-1$ & $1-1-3$ & $3-2-3$ & \\
6 & $1-0-1$ & $1-1-2$ & $2-2-3$ & \\
7 & $0-0-1$ & $0-1-1$ & $1-1-3$ & \\
8 & $0-0-1$ & $0-1-1$ & $1-1-2$ & \\
9 & & $0-0-1$ & $1-0-2$ & \\
10 & & & $0-0-2$ & \\
11 & & $0-0-1$ & \\
\hline
\end{tabular}

\section{Tetrabamato}

Para algunos autores el tetrabamato o carbamato-fenobarbital es considerado como el fármaco más indicado para tratamientos ambulatorios, o SAA leves o moderados. Presenta un menor índice adictivo y permite una menor vigilancia. Se utiliza a dosis entre 6 y 12 comprimidos de 300 mg./día, dependiendo del grado de sintomatología de abstinencia, con reducción progresiva de un comprimido/día. Sólo es posible su administración por vía oral (Tabla IV). Debe evitarse su administración en pacientes con alteración de la función hepática.

\section{Benzodiacepinas}

Las benzodiacepinas provocan una estimulación de la actividad GABAérgica, incrementan la afinidad por los receptores GABAA, bloquean la hiperactividad noradrenérgica, disminuyen la sintomatología relacionada directamente con el incremento de corticoesteroides, y la probabilidad de aparición de crisis epilépticas ${ }^{29}$.

Las benzodiacepinas están consideradas en los estudios de investigación y de consenso como el fármaco de elección para el tratamiento del síndrome de abstinencia en EEUU $^{30}$. En la revisión y meta-análisis realizados por Moskowitz se concluyó que "las ben- zodiacepinas deben constituir el tratamiento de elección frente a cualquier otra terapéutica" ${ }^{31}$. También las considera de elección Ozdemir $^{32}$. No existe ninguna benzodiacepina que se considere de eficacia superior al resto y todos los estudios han demostrado su eficacia frente al placebo en la reducción de los signos y síntomas de la abstinencia ${ }^{33,34}$. La inconveniencia de la utilización de dichos agentes es el potencial de abuso, el detrimento del nivel cognitivo y la desinhibición, y su selección debe estar determinada por las condiciones de cada paciente, tales como la edad o la presencia de una enfermedad hepática, o la historia de crisis convulsivas. Constituye una ventaja el hecho de la posiblidad de su administración por vía sublingual o endovenosa. Destacar que un apreciable porcentaje de los pacientes con dependencia del alcohol toman benzodiacepinas (un 24\%-50\% en un estudio nórdico), y en éstos el riesgo de dependencia a estos fármacos no se ha estudiado profundamente. En algunos estudios se sugiere que el riesgo de dependencia de las benzodiacepinas constituye el $2 \%$ de los pacientes con dependencia del alcohol ${ }^{35}$.

Clásicamente se prefería el diacepam o el clordiacepóxido para el tratamiento de la abstinencia, aunque actualmente recientes revisiones abogan por el uso de benzodiacepinas de vida media corta o intermedia como el oxacepam o el loracepam respectivamente, 
Tabla IV. Pautas de tetrabramato

\begin{tabular}{lcccc}
\hline Día & Pauta 1 & Pauta 2 & Pauta 3 & Pauta 4 \\
\hline 1 & $2-2-2$ & $2-2-3$ & $3-3-4$ & $4-4-4$ \\
2 & $2-1-2$ & $2-2-2$ & $3-3-3$ & $4-3-4$ \\
3 & $1-1-2$ & $2-1-2$ & $3-3-3$ & $3-3-4$ \\
4 & $1-1-1$ & $1-1-2$ & $3-2-3$ & $3-3-3$ \\
5 & $1-0-1$ & $1-1-1$ & $2-2-3$ & $3-3-3$ \\
6 & $0-0-1$ & $1-0-1$ & $2-2-2$ & $3-2-3$ \\
7 & & $0-0-1$ & $2-1-2$ & $2-2-3$ \\
8 & & & $1-1-2$ & $2-2-2$ \\
9 & & & $1-1-1$ & $2-1-2$ \\
10 & & & $1-0-1$ & $1-1-2$ \\
11 & & & $0-0-1$ & $1-1-1$ \\
12 & & & & $1-0-1$ \\
13 & & & & $0-0-1$
\end{tabular}

Tabla V. Benzodiacepinas en el síndrome de abstinencia alcohólica

\begin{tabular}{|c|c|c|c|c|}
\hline Fármaco & $\begin{array}{l}\text { Dosis } \\
\text { inicial }\end{array}$ & $\begin{array}{c}\text { Inicio de } \\
\text { los efectos }\end{array}$ & $\begin{array}{l}\text { Duración de } \\
\text { los efectos }\end{array}$ & $\begin{array}{c}\text { Semivida } \\
\text { (horas) }\end{array}$ \\
\hline \multicolumn{5}{|l|}{ Vida corta } \\
\hline Loracepam & $1-5 \mathrm{mg} . / 6 \mathrm{hr}$. & Intermedio & Intermedia & $10-20$ \\
\hline Oxacepam & 30-120 mg./6 hr. & Lento & Corta & $3-21$ \\
\hline \multicolumn{5}{|l|}{ Vida prolongada } \\
\hline Clordiacepóxido & $25-100 \mathrm{mg} . / 6 \mathrm{hr}$. & Intermedio & Prolongada & $5-30$ \\
\hline Diacepam & 4-20 mg./6 hr. & Rápido & Prolongada & $20-50$ \\
\hline
\end{tabular}

Tabla VI. Tipos de tratamiento con benzodiacepinas ${ }^{63}$

\begin{tabular}{|c|c|c|c|}
\hline & Vía & Diacepam & Clordiacepóxido \\
\hline Pauta de 4 días & Oral & $\begin{array}{l}\text { Día 1: } 20 \text { mg./6 hr. } \\
\text { Día 2: } 20 \mathrm{mg} . / 8 \mathrm{hr} \text {. } \\
\text { Día 3: } 20 \mathrm{mg} . / 12 \mathrm{hr} \text {. } \\
\text { Día 4: } 20 \mathrm{mg} . / 24 \mathrm{hr} \text {. }\end{array}$ & $\begin{array}{l}\text { Día 1: } 100 \mathrm{mg} . / 6 \mathrm{hr} . \\
\text { Día 2: } 100 \mathrm{mg} . / 8 \mathrm{hr} . \\
\text { Día 3: } 100 \mathrm{mg} . / 12 \mathrm{hr} \text {. } \\
\text { Día 4: } 100 \mathrm{mg} . / 24 \mathrm{hr} .\end{array}$ \\
\hline Método de carga & $\begin{array}{c}\text { Oral } \\
\text { Intravenosa }\end{array}$ & $\begin{array}{c}20 \text { mg. cada 1-2 horas } \\
\text { hasta sedación. } \\
20 \text { mg. inicialmente, } \\
\text { después una infusión de } \\
20 \text { mg./hr. hasta sedación. }\end{array}$ & $\begin{array}{c}100 \text { mg. cada } 2 \text { horas } \\
\text { hasta sedación. } \\
\text { No recomendado. }\end{array}$ \\
\hline $\begin{array}{l}\text { Tratamiento del } \\
\text { delirium tremens }\end{array}$ & Intravenosa & $\begin{array}{l}20 \text { mg. inicialmente, } \\
\text { después } 5 \text { mg. cada 5-10 } \\
\text { min. hasta sedación. }\end{array}$ & No recomendado. \\
\hline
\end{tabular}




\section{Tabla VII. Regimenes específicos terapéuticos. Protocolo para la desintoxicación del alcohol mediante dosis elevadas de benzodiacepinas. Adaptado de Shaw (1995) ${ }^{64}$.}

Altas dosis de benzodiacepinas en los días 1-3:

Diacepam 40 mg. o más, loracepam 8 mg. o más.

Reducción de la dosis de aprox. el 25\% diario.

En casos severos: diacepam 10-20 mg. cada hora hasta

alcanzar una adecuada sedación.

Reducción de dosis de un $25 \%$ diariamente.

siendo muy escasamente utilizado el alprazo$\operatorname{lam}^{36}$. Las benzodiacepinas de semivida larga como el diacepam o el clordiacepóxido, presentan la ventaja de un menor efecto rebote de los síntomas y una acción más prolongada. Sin embargo, presentan los inconvenientes de un mayor riesgo de acumulación y sedación excesiva en algunos grupos de pacientes incluyendo aquellos de edad avanzada o que presentan una enfermedad hepática, y una absorción errática por vía intramuscular. Se metabolizan principalmente por vía hepática, mediante oxidación o conjugación y poseen metabolitos activos de larga vida media. El diacepam y su metabolito el desmetildiacepam presentan un tiempo de vida media entre 30 y 90 horas respectivamente. Esto permite un SAA relativamente libre de variaciones y disminuye la necesidad de administrar dosis adicionales. En caso de aparición de convulsiones el diacepam es la benzodiazepina de elección, ya que presenta un rápido comienzo de acción y mayor capacidad anticonvulsivante que el clordiacepóxido. Por otro lado, el cloridazepóxido tiene la ventaja de ser más seguro si se administra conjuntamente con el alcohol, y parece tener menor potencial de abuso.

Las benzodiacepinas de semivida corta o intermedia como el oxacepam o el loracepam presentan un menor riesgo de sedación y de acumulación, no tienen metabolitos activos y son especialmente útiles en ancianos y en pacientes con enfermedad hepática. El loracepam en concreto se metaboliza por fase II (conjugación), sin utilizar el citocromo P450.
Sin embargo producen con mayor frecuencia sintomatología de rebote.

En la mayoría de estudios las benzodiacepinas son administradas en dosis fijas, por ejemplo $50 \mathrm{mg}$. de clordiacepóxido cada 6 horas durante 24 horas y posteriormente 25 mg. cada 6 horas durante 48 horas; diacepam $10 \mathrm{mg}$. cada 6 horas durate 24 horas y $5 \mathrm{mg}$. cada 6 horas durante 48 horas o loracepam 2 mg. cada 6 horas durante 24 horas, con reducción a $1 \mathrm{mg}$. cada 6 horas durante 48 horas. Otra pauta sería la de administrar diacepam a dosis de entre 10 y $30 \mathrm{mg}$./día, con reducción progresiva en los siguientes 10-15 días, en los casos leves o bien ante la sintomatología de abstinencia severa $20 \mathrm{mg}$. de diacepam o equivalente cada 2 horas hasta la desaparición de la sintomatología, sin sobrepasar la dosis diaria de 100 mg. ("método de carga"), con reducción de un $20 \%$ diario ${ }^{37}$. Una alternativa es administrar la medicación dependiendo de los síntomas en una dosis variable. En estos casos el paciente ha de ser valorado mediante escalas, iniciando el tratamiento si se supera un determinado umbral de severidad ${ }^{38,39}$. Se ha demostrado que son tan eficaces las pautas de tratamiento que varían según la sintomatología de abstinencia del paciente como las pautas fijas, con la ventaja en el primer caso de que se administra menor dosis de tratamiento y durante un menor periodo de tiempo ${ }^{40}$. Cuando la puntuación en la CIWA-Ar es superior o igual a 810, administrar clordiacepóxido 50-100 mg, diacepam 10-20 mg. o loracepam 2-4 mg. cada hora hasta control de la sintomatología. 
Esta es una actuación segura y con escasa aparición de efectos secundarios.

\section{Carbamacepina y otros agentes anticon- vulsivantes.}

La carbamacepina actúa sobre diversos sistemas neurotransmisores incluyendo GABA y noradrenalina, pero su mecanismo de acción en el SAA es desconocido. Ha sido muy utilizada desde los años 60 en Escandinavia. La carbamacepina se utiliza ampliamente en Europa en el síndrome de abstinencia de alcohol, se considera superior al placebo y similar en eficacia a las benzodiacepinas en la abstinencia leve o moderada ${ }^{41}$. En los años 70 se utilizó como tratamiento del delirium tremens en combinación con otros fármacos sedantes ${ }^{42}$. No existen sin embargo, datos sobre su eficacia en la prevención de delirium o convulsiones en humanos, aunque sí en animales. No provoca depresión respiratoria ni alteraciones cognitivas, tampoco posee potencial de abuso, ni interacción con el alcohol. Se cree que podría retardar el fenómeno de sensibilización o "kindling"43. En cualquier caso, no se recomienda como monoterapia, si no en combinación con benzodiacepinas. Presenta como inconvenientes la aparición de efectos secundarios en un $10 \%$ de los casos en forma de vértigo, náuseas, prurito, ataxia, cefalea y xerostomía ${ }^{44}$. Es potencialmente tóxico en asociación con algunos antibióticos, antidepresivos y otros anticonvulsivantes ${ }^{45}$.

La fenitoina ha sido utilizada conjuntamente con el clordiacepóxido para prevenir la aparición de convulsiones. Sin embargo, se considera que las benzodiacepinas son en la mayoría de los casos suficientes para prevenirlas.

El ácido valproico, la gabapentina y la vigabatrina han demostrado asímismo ser más útiles que el placebo en el tratamiento del SAA ${ }^{46}$.

\section{Agonistas $\alpha$ adrenérgicos}

La clonidina es un agonista parcial $\alpha 2$ adrenérgico. Fue estudiada en el SAA debido a que se conocía la eficacia de este fármaco en el tratamiento del síndrome de abstinencia a los opiáceos ${ }^{47}$. La dosis es de 0,2 mg. cada 8 horas, y ha demostrado su eficacia en la reducción de la sintomatología de abstinencia leve o moderada, sobretodo sobre el incremento de la frecuencia cardiaca o la tensión arterial, incluso igual a la del clordiacepóxido en un estudio realizado en 1987 por Baumgarten y Rowen ${ }^{48}$. No tiene efectos anticonvulsivos o antidelirium. Se utiliza de forma conjunta con benzodiacepinas. Precisa de una adecuada monitorización de la tensión arterial.

\section{Antagonistas $\beta$ adrenérgicos}

Son escasamente utilizados en nuestro medio. Estos fármacos (propranolol y atenolol) son especialmente útiles en la reducción de las manifestaciones autonómicas del síndrome de abstinencia, y su uso se propuso por primera vez en el Lancet (Editorial, 1973). No existe ninguna evidencia de que estos agentes sean útiles en la prevención de convulsiones o delirium, e incluso pueden enmascarar su aparición, debido a la reducción de la sintomatología autonómica ${ }^{49}$. Podrían ser de interés en aquellos pacientes con síntomas no graves de abstinencia y en asociación con benzodiacepinas. Según Kraus ${ }^{50}$, los pacientes que recibieron atenolol durante un ingreso presentaron una reducción del tiempo de hospitalización y necesitaron menos benzodiacepinas. Hay que tener cuidado en pacientes diabéticos, pues puede enmascarar la sintomatologia de la hipoglucemia, y en aquellos con enfermedad pulmonar o miocardiopatía.

\section{Agentes neurolépticos}

Las fenotiazinas y las butirofenonas han demostrado alguna eficacia en la reducción de los signos y síntomas de la abstinencia, pero no previenen la aparición de crisis convulsivas y son menos eficaces que las benzodiacepinas en la prevención de la aparición del delirium. Se utilizan fundamentalmente junto con las benzodiacepinas como tratamiento del 
paciente agitado o que presenta alucinaciones, como mencionaremos a continuación.

\section{Magnesio}

Los niveles de magnesio se reducen durante el síndrome de abstinencia, normalizándose al finalizar éste. Se ha intentado tratamiento parenteral con magnesio asociado a benzodiacepinas, no habiéndose obtenido diferencias en la gravedad de la sintomatología de abstinencia, la prevención de convulsiones o delirium ${ }^{51}$. Solamente estaría indicado en el caso de arritmias cardíacas o alteraciones neurológicas secundarias a un déficit de magnesio.

\section{Alcohol etílico}

La administración de alcohol previene los síntomas de abstinencia, y aunque hay estudios que describen su administración, no existe ninguno que valore de forma controlada su seguridad o eficacia. No puede ser aceptado su uso en el tratamiento del síndrome de abstinencia por múltiples razones, entre ellas el planteamiento de la deshabituación-rehabilitación del paciente con dependencia del alcohol, y su toxicidad.

El Litio demostró ser más útil que el placebo en el control de los síntomas de abstinencia al alcohol a dosis de 900 mg./día, en el estudio realizado por Sellers en $1976^{52}$. Este autor postulaba que el litio disminuiría la actividad de la bomba sodio-potasio ATPasa, que se incrementa durante el SAA.

Según Borg y Weinholt en 1982, la bromocriptina a dosis de $7.5 \mathrm{mg}$. también resultó útil comparado con el placebo en el tratamiento del SAA de 8 pacientes, con un efecto significativo sobre la ansiedad, el temblor, las náuseas y la sudoración ${ }^{53}$. Según Burroughs $^{54}$ su eficacia es inferior a la del clormetiazol o el clordiacepóxido.

El ácido gamma-hidroxibutírico (GHBA) es una sustancia endógena con propiedades sedantes, cuyo mecanismo de acción es desconocido. Ha demostrado su eficacia en la reducción de la sintomatología de la abstinencia al alcohol, pero presenta un número importante de efectos secundarios, especialmente vértigo ${ }^{55}$.

Los antagonistas del calcio como el verapamilo, se han utilizado en el tratamiento de la abstinencia grave del alcohol, aunque aún no se han realizado ensayos a doble ciego. No es utilizado en nuestro medio ${ }^{56}$.

Los inhibidores selectivos de la recaptación de serotonina pueden aliviar el SAA, pero no existen evidencias de su utilidad ${ }^{57}$.

\section{Abstinencia grave del alcohol. Delirium Tremens.}

Se trata de una situación clínica grave que requiere la hospitalización inmediata, debido a la alta mortalidad que conlleva y a la incapacidad del paciente para su autocuidado. Se han identificado algunos factores en un estudio realizado por Palmstierna ${ }^{58}$, que pueden contribuir al riesgo de padecer un delirium tremens, como son una infección u otra enfermedad médica intercurrente, frecuencia cardiaca por encima de 120 pulsaciones/ minuto en el momento de la admisión, signos de abstinencia con niveles de alcoholemia superiores a $1 \mathrm{gr}$./litro, una historia de crisis convulsivas y una historia de delirium. Normalmente en estos casos la puntuación en la escala CIWA-Ar es igual o superior a 20. El tratamiento debe ser administrado por vía endovenosa, debe poseer propiedades sedativas y antiepilépticas, y estabilizar las funciones neurovegetativas. Se requieren asimismo una buena hidratación con sueroterapia, alternando suero fisiológico y suero glucosado, manteniendo la especial precaución de administrar previamente tiamina, por el riesgo si existe hipoglucemia de encefalopatía de Wernicke. Igualmente importante es mantener el equilibrio iónico, vigilando especialmente el riesgo de hipokaliemia. Es conveniente mantener al paciente en un entorno que favorezca la estimulación sensorial y la orientación. El paciente debe sujetarse de forma mecánica si existe agitación, adminis- 
trando neurolépticos sedantes si ésta es importante y no cede con el tratamiento habitual. Se administrará clormetiazol $150 \mathrm{ml}$. a un ritmo de 100 microgotas/minuto, hasta conseguir una sedación superficial y continuando posteriormente con dosis de 10-20 microgotas/minuto, valorándose frecuentemente el estado de conciencia debido al riesgo de depresión respiratoria. Es igualmente eficaz utilizar diacepam por vía intravenosa 20 mg. lenta inicialmente, y posteriormente 5 mg. cada 5-10 minutos hasta la sedación. El clordiacepóxido no está indicado como tratamiento del delirium tremens. Si persiste la agitación, se recomienda añadir $25 \mathrm{mg}$. de levomepromazina por vía i.m. o bien 300 mg. de tiaprida vía i.m. Algunos autores preconizan el uso de carbamacepina 300-600 mg./día vía oral. El paciente debe monitorizarse clínica y analíticamente, y han de tratarse de forma enérgica la aparición de complicaciones, como infecciones intercurrentes y alteraciones electrolíticas o metabólicas.

\section{Tratamiento de las convulsiones}

Las convulsiones por lo general ocurren en pacientes no tratados, y pueden preceder a los otros síntomas de abstinencia. Más de un tercio de los pacientes que presentan convulsiones y no reciben tratamiento desarrollarán un cuadro de delirium tremens. En pacientes sin historia de crisis convulsivas, las benzodiacepinas o el clormetiazol son el tratamiento de elección para prevenir la aparición de éstas. Sin embargo, en pacientes con antecedentes de crisis epilépticas se aboga por la utilización conjunta de benzodiacepinas y un fármaco antiepiléptico como la fenitoína o el ácido valproico y en menor grado la gabapentina. Por regla general aparece una sola crisis convulsiva, lo que no precisa tratamiento. Si aparece una segunda crisis se aconseja instaurar tratamiento, recomendándose aumentar la dosis de clormetiazol o de benzodiacepinas, o añadir diacepam o loracepam. Si las crisis se presentan de forma continua se recomienda el uso de diacepam 5-10 mg. vía i.v. a pasar de forma lenta, repetibles cada 5 minutos hasta alcanzar los $25 \mathrm{mg}$. o el control del cuadro. En algunos estudios se considera al loracepam como la droga de primera línea para la profilaxis y tratamiento de las crisis convulsivas ${ }^{59}$.

\section{Situaciones especiales en el tratamiento del síndrome de abstinencia.}

Finalmente, es preciso hacer mención al tratamiento del síndrome de abstinencia en algunos grupos de población especiales como son los adolescentes, los ancianos, la mujer embarazada y el paciente con enfermedades médicas comórbidas. En cuanto a los adolescentes no existen estudios que recomienden un tratamiento diferente que el analizado en la población adulta general.

En los ancianos, habrá que tener en cuenta la mayor gravedad de la sintomatología de abstinencia, siendo mayor el riesgo de convulsiones o delirium, aunque existen algunas publicaciones recientes que indican que la gravedad de la sintomatología de abstinencia no es dependiente de la edad ${ }^{60}$. Los pacientes ancianos que presenten síntomas de abstinencia deben ser hospitalizados, a no ser que tengan un óptimo soporte socio-familiar, que la sintomatología sea leve y que no presenten antecedentes de complicaciones de un síndrome de abstinencia, y en ausencia de enfermedad comórbida. No existen estudios en esta población, sin embargo es recomendable la administración de benzodiacepinas de tiempo de vida media corto o intermedio, a fin de prevenir el mayor riesgo de acumulación y secundariamente de sedación, si bien son menos eficaces para prevenir convulsiones y pueden provocar síntomas de rebote, por lo que la dosis deberá ser monitorizada con mayor intensidad.

En la mujer embarazada no existen estudios acerca de cuál es el manejo terapéutico idóneo. Si bien existe el riesgo teratógeno asociado a la utilización de benzodiacepinas, las consecuencias asociadas a la presencia de un síndrome de abstinencia severo pueden ser fatales tanto para el feto como para 
la madre, recomendándose el uso de benzodiacepinas a las dosis mínimas necesarias para controlar la sintomatología, especialmente loracepam.

En los pacientes con enfermedades comórbidas hepáticas, coronarias, pulmonares o con diabetes, existe un mayor riesgo de que la sintomatología sea más intensa y de aparición de complicaciones graves, debiendo utilizarse farmacoterapia preventiva siempre o ante la presencia de sintomatología de abstinencia leve. En el paciente post-quirúrgico deben tratarse también los síntomas de abstinencia utilizando la terapéutica desarrollada en este capítulo, si bien debe ser orientada a la prevención y tratamiento de las manifestaciones autonómicas y muy especialmente a la prevención de alteraciones psicomotrices, consiguiendo una adecuada sedación, siempre bajo una estricta monitorización. Las pautas de dosificación serán normalmente más duraderas en estos casos que las habitualmente utilizadas en unidades de desintoxicación ${ }^{61}$.

\section{REFERENCIAS BIBLIOGRÁFICAS}

(1) Buschbaum DG, Buchanan RG, Poses RM, Schnoll SH, Lawton MJ. Physician detection of drinking problems in patients attending a general medical practice. J Gen Intern Med. 1992;7:517-521.

(2) Cirera E y cols. Alcoholismo en el Hospital General. Med. Clin 1985;85: 96-98.

(3) Michael F. Mayo-Smith, MD, MPH; for the American Society of Addiction Medicine Working Group on Pharmacological Management of Alcohol Withdrawal. Pharmacological Management of Alcohol Withdrawal. Jama 1997;144151.

(4) Victor M., Adams R. The effect of alcohol on the nervous system. Research publication of the Association for Nervous and Mental Disorders. 1953; 32:526-573.

(5) Stockwell T, Sitharthan T, McGrath D. The measurement of alcohol dependence and impaired control in the general population. Addiction 1994; 89: 167-174.
(6) Foy A, Kay J. The incidence of alcohol-related problems and the risk of alcohol withdrawal in a general hospital population. Drug Alcohol Rev. 1995; 14:49-54.

(7) Nevo I, Hamon M. Neurotransmitter and neuromodulatory mechanisms involved in alcohol abuse and alcoholism. Neurochem Int 1995;26,4:305-306.

(8) Kumari M. Ticku MK. Regulation of NMDA receptors by ethanol. Progress in Drug Research. 2000;54:152-189.

(9) Casas M, Prat G, Guardia J. Los efectos del etanol sobre el sistema de neurotransmisión dopaminérgico como posible sustrato biológico de los trastornos psíquicos observados en el alcoholismo. En: Casas M, Gutiérrez M, San L. Psicopatología y alcoholismo. Ediciones en Neurociencias. Barcelona, 1994.

(10) Heinz A, Scmidt K, Baumm SS. Influence of dopaminergic transmission on severity of withdrawal syndrome in alcoholism. J Stud Alcohol 1996; 57:471-474.

(11) Ballenger J.C., Post R.M. kindling as a model for the alcohl withdrawal syndromes. Br. J. Psychiatry. 1978;133:1-14.

(12) Hundt W. Zimmermann U. Potting M. Spring K. Holsboer F. The combined dexamethasonesuppresion/crh-stimulation test in alcoholics during and after acute withdrawal. Alcoholism: Clinical and Experimental Research. 2001;25 (5):687-691.

(13) Nutt D, Adinoff B. Linnoila M. Benzodiacepines in the treatment of alcoholism. In Recent Development in Alcoholism. Galanter M. ed. 1989; pp:283-313.

(14) Soler Insa P.A., Sanahuja Solans J., Mengual Prims A. Trastornos mentales y del comportamiento debidos al consumo de alcohol. Tratado de psiquiatría. Editor: Barcia Salorio. 2000;pp 221-242.

(15) Guerola J, Martínez C. Urgencias médicas originadas por el consumo de alcohol. Tratado de Alcohología. Ed.:Cuevas J y Sanchís M. 2000. pp.: 305-357.

(16) Ferguson JA, Suelzer CJ, Eckert GJ. Risk factors for delirium tremens development. J Gen Intern Med 1996; 11: 410-414.

(17) Williams D, Lewis J, McBride Andrew. A comparison of rating scales for the alcohol withdrawal syndrome. Alcohol and Alcoholism. 2001. Vol 36, No 2, pp:104-108. 
(18) Hugh Myrick, MD, Raymond F. Anton, MD. Clinical Management of Alcohol Withdrawal. CNS Spectrums 2000;5(2):22-23.

(19) Saitz R, O'Malley SS. Pharmacotherapies of alcohol abuse: withdrawal and treatment. Med Clin North Am. 1997;81:881-907.

(20) G.K. Shaw, S. Waller, C.J. Latham, G. Dunn, A.D. Thomson. The detoxification experience of alcoholic in-patients and predictors of outcome. Alcohol and alcoholism. 1998. Vol. 33. NO.3,pp:291-303.

(21) Abbot JA, Quinn D, Knox L. Ambulatory medical detoxification for alcohol. Am J Drug Alcohol Abuse. 1995;21:549-563.

(22) Hyashida M, Alterman Al, McClellan AT, et al: Comparative effectivenes and costs of inpatient and oupatient detoxification of patients with mild to moderate withdrawal syndrome. N Eng J Med. 1989;320:358-365

(23) Pattison Me: Management of alcoholism in medical practice. Med Clin North Am. 1977;61: 797-809.

(24) Withfield CL: Nondrug detoxification in Phenomenology and Treatment of Alcoholism. Withfield C. New York, Spectrum Press. 1980.

(25) Gallant DM: Reduction of ethanol intake by pharmacologic agents-investigational problems. Alcohol Clin Exp Res. 1992;16:472-473.

(26) Shaw JM, Kolesar GS, Sellers EM, Kaplan HL, Sandors P. Development of optimal treatment tactics for alcohol withdrawal, Y: assesment and effectiveness of supportive care. J Clin Psychopharmacol. 1981;382-387.

(27) Myrick Hugh, MD, Anton RF, MD. Clinical Management of Alchol withdrawal. CNS Spectrums 2000;5(2):22-32.

(28) Glatt, M., George, H.R., Frisch, E.P. Controlled study of chlormethiazole in treatment of the alcohol withdrawal phase. British Medical Journal.1965;2:401-404.

(29) Lejoyeux Michel, Solomon Jacquelyn, Adès Jean. Benzodiazepine Treatment for alcoholdependent patients. Review. Alcohol and alcoholism. 1998; 33:563-575.

(30) Institute of Medicine. Prevention and Treatment of Alcohol Problems. Washington, DC: National Academy Press; 1990:268-269.

(31) Moskowitz G, Chalmers TC, Sacks HS, Fagerstrom RM, Smith H. Deficiencies of clinical trials of alcohol withdrawal. Alcohol Clin Exp Res. 1983;7:42-46.
(32) Ozdemir V, Bremmer KE, Naranjo CA. Treatment of alcohol withdrawal syndrome. Trends in Clinical Practice. Anals of Medicine. 1993;26:101-105.

(33) Sellers EM, Naranjo CA, Harrison M, Devenyl P, Roach C, Sykora K. Diacepam loading: simplified treatment of alcohol withdrawal. Clin Pharmacol Ther. 1983;34:822-826.

(34) Adinoff B. Double blind study of alprazolam, diacepam, clonidine and placebo in the alcohol withdrawal syndrome: preliminary findings. Alcohol Clin Exp Res. 1995;18:873-878.

(35) Borg S., Carlsson S., Lafolie P. Benzodiazepine/ alcohol dependence and abuse. Benzodiazepine Dependence, Hallström,C.ed. 1993, pp:119-127.

(36) Gallant DM. Improvements in treatment of alcohol withdrawal syndromes. Alcohol Clin Exp Res. 1989;13:721-722.

(37) Rojo Rodes J.E., Cirera Costa E. Interconsulta psiquiátrica. Ed. Masson. 1977,pp:539-545.

(38) Wartenberg AA, Nirenberg TD, Liepman MR, Silvia LY, Begin AM, Monti PM. Detoxification of alcoholics:improving care by symptomtriggered sedation. Alcohol Clin Exp Res. 1990;14:71-75.

(39) Sullivan JT, Swift RM, Lewis DC. Benzodiazepine requierements during alcohol withdrawal syndrome: clinical implications of using a standarized withdrawal scale. J Clin Psychopharmacol. 1991; 11:291-295.S

(40) Saitz R, Mayo-Smith MF, Robers MS, Redmond HA, Bernard DR, Calkins DR. Individualized treatment for alcohol withdrawal: a randomized double-blind controlled trial. JAMA. 1994;272: 519-523.

(41) Bjorkquist SE, Isohanni M, Makella R, Malinen $\mathrm{L}$. Ambulant treatment of alcohol withdrawal symptoms with carbamazepine: a formal multicentre double-blind comparison with placebo Acta Psychiatr Scand. 1976;53:333-342.

(42) Brune, F., Busch, H. Anticonvulsivant-sedative treatment of delirium alcoholism. Quarterly Journal of Studies on alcohol. 1971;32:334-342.

(43) Ballenger JC, Post RM. kindling as a model for the alcohol withdrawal syndrome. $\mathrm{Br} J$ Psychiatry. 1978;133:1-4.

(44) Butler, D., Messiah, F.S. Alcohol withdrawal and carbamazepine. 1986;3:113-129.

(45) Ketter, T.A., Post, R.M., Worhington K. Principles of clinically important drug interactions with 
carbamacepina. Journal of Clinical Psycopharmacology. 1991;11:19-203.

(46) Malcolm R. Myrick H. Brady KT. Ballenger JC. Update on anticonvulsivants for the treatment of alcohol withdrawal. American Journal on Addictions. 2001, 10 Suppl:16-23.

(47) Bjorkvist, S.E. Clonidine in alcohol withdrawal. Acta Psychiatrica Scandinavica. 1975;52:256263.

(48) Baumgartner GR, Rowen RC. Clonideine vs chlordiazepoxide in the management of acute alcohol withdrawal syndrome. Arch Intern Med. 1987;147:1223-1226.

(49) Zilm DH, Jacob MS, Mac Leod SM, Sellers EM, Ti TY. Propranolol and chlordiazepoxide effects on cardiac arrhytmias during alcohol withdrawal. Alcohol Clin Exp Res. 1980;4:400405.

(50) Kraus ML, Gottlieb LD, Horwitz RI, Anscher M. Randomized clinical trial of atenolol in patients with alcohol withdrawal. N Engl J Med. 1985; 313:905-910.

(51) Wilson A, Vulcano B. A double-blind placebocontrolled trial of magnesium sulfate in the ethanol withdrawal syndrome. Alcohol Clin Exp Res. 1984;8:542-545.

(52) Sellers, EM. Cooper, SD. Zilm DH. Shanks C. Lithium treatment during alcohol withdrawal. Clinical Pharmacology and Therapy. 1976;20: 199-206.

(53) Borg, V. Weinholt, T. Bromocriptine in the treatment of alcohol withdrawal syndrome. Acta Psychiatrica Scandinavica. 1982;65:101-111.

(54) Burroughs AK, Morgan MY, Sherlock S. Double blind controlled trial of bromocriptine, chlordiazepoxide and chlormetiazole for alcohol withdrawal symptoms. Alcohol and alcoholism. 1985; 20:263-271.

(55) Gallimberti, L., Canton, G., Gentile, N., Ferri, M., Cibin, M., Ferrara, S.D., Fadda, F. and Gessa, G.L. Gamma-hydroxy-butyric acid for treatment of alcohol withdrawal syndrom. Lancet. 1989 ii,787-789.

(56) Roy-Byrne PP, Ward NG, Donnell PJ. Valproate in anxiety and withdrawal syndromes. J. Clin. Psychiatry. 1989; 50: 44-48.

(57) Thompson MD. Gonzalez N. Nguyen T. Comings DE. George SR. O'Dowd BF. Serotonin transporter gene polymorphisms in alcohol dependence. Alcohol. 2000;22:61-67.

(58) Palmstierna T. A model for predicting alcohol withdrawal delirium. Psychiatric Services. 2001;52:820-823.

(59) Ahmed S, Chadwick D, Walker RJ. The management of alcohol related seizures: an overview. Hosp Med 2000 Nov;61:793-796.

(60) Tilman Wetterling, Martin Driessen, Rolf-Dieter Kanitz, Klaus Junghanns. The severity of alcohol withdrawal is not age dependent. Alcohol and Alcoholism. Vol 36, No 1, pp. 7578, 2001.

(61) Spies CD, Rommelspacher H. Alcohol withdrawal in the surgical patient: prevention and treatment. Anesth Analg 1999 Apr;88(4): 946-954.

(62) Soler PA, Gascón J. Protocolos de terapéutica psiquiátrica. 1995. pp: 157-164.

(63) Soler PA, Gascon J. Recomendaciones terapéuticas en los trastornos mentales. De Masson. 1999. pp:11-25.

(64) Shaw,GK. Detoxification: the use of benzodiazepines. Alcohol and Alcoholism. 1995;30:765-770. 